\title{
On the existence of constant scalar curvature Kähler metric: a new perspective
}

\author{
Xiuxiong Chen \\ In memory of Prof. Weiyue Ding
}

June 23, 2015

\section{Introduction}

In 1950s, E. Calabi first proposed to study the constant scalar curvature Kähler (cscK) metric problems. His ideal is to find the best canonical metric in each given Kähler class (cf. [14, 15), which results in a 4th order, fully nonlinear partial differential equation. When the first Chern class has a definite sign (positive, negative or zero), the cscK metric in the suitable multiple of the first Chern class reduces to a Kähler-Einstein metrics. Calabi's program on Kähler-Einstein metrics is the center of the field for the last few decades where all efforts and techniques of many mathematicians are devoted to, leading to the final resolution of this difficult problem. With the existence problem of Kähler-Einstein metric settled eventually, perhaps it is time to discuss how to attack Calabi's original problem in full generality. In this note, we propose a "new, " continuity path in a given Kähler class to solve the cscK metric problem. Module out the profound difficulty in analysis, we hope this will shed light on the existence problem from direct PDE approach. This will largely be an expository paper where we concentrate on explaining various aspects of this new path, except Theorem 1.8 in which we proved openness along this proposed path. Perhaps more intriguely, while we can not prove that the K-energy functional is cocercive in terms of geodesic distance if there exists a cscK metric, we can prove that for any $t \in(0,1)$, this family of twisted K-energy is indeed coercive in terms of geodesic distance (cf. Theroem 3.4 for more precise statement). While we wish this path were new, as usual, we find "footsteps of others", in particular the works of Fine, Stoppa and Lejmi-Székelyhidi [56, 77, 64. The openness theorem is inspired by the renown work of LeBrun-Simanca 62] on deformation of extremal Kähler metrics.

\subsection{A brief account on Kähler-Einstein problems}

In 1976, S.-T. Yau solved the famous Calabi conjecture (by showing the existence of Ricci flat Kähler metric) if $C_{1}=0$. Around the same time, S.-T. Yau and T. Aubin independently solved the existence of the Kähler-Einstein metric if $C_{1}<0$. In 1990, S.-T. Yau first suggested that the existence of KählerEinstein metric is related to certain notion of stability of the underlying polarization. In 1997, G. Tian introduced the so-called K-stability (via special degeneration) and showed that the existence of Kähler-Einstein metric necessarily implies the K-stability of the underlying polarization through special degeneration. In 2002, S. K. Donaldson reformulated it into a notion of algebraic K-stability. In 2012, with S. K. Donaldson and S. Sun, we proved

Theorem 1.1 (Chen-Donaldson-Sun[25, 26, 27]) K-stable Fano manifolds admit Kähler-Einstein metrics. 
The converse part of the above theorem is due to work of G. Tian 84], S. K. Donaldson[47, Stoppa [76], and the most general form is due to R. Berman [5]. There is also a Ricci flow approach to attack the existence problem of the Kähler-Einstein metrics. The fundamental problem in the Kähler Ricci flow is the so-called Hamilton-Tian conjecture: for any sequence of time $t_{i} \rightarrow \infty$, the corresponding sequence of Kähler metrics converge in Gromove-Hausdorff sense to a Kähler Ricci soliton with at most codimension 4 singularities. In 2014, Chen and Wang give an affirmative answer to this conjecture.

Theorem 1.2 (Chen-Wang[33]) For any Fano manifold, the Kähler Ricci flow will always converge to a Kähler-Ricci soliton with at most codimension 4 singularities in the sense of Cheeger-Gromov. Moreover, the complex structure may jump in the limit.

While there are many following up problems related to the existence problem of Kähler-Einstein metrics, the central problem in Kähler geometry is to solve the more general conjecture on the existence of cscK metrics.

Conjecture 1.3 (Yau-Tian-Donaldson) The underlying polarized Kähler manifold $(M,[\omega])$ is $K$ stable if and only if there exists a constant scalar curvature Kähler metric in $[\omega]$.

\subsection{A new continuity path}

For any Kähler manifold $(M,[\omega])$, consider the space of Kähler potentials

$$
\mathcal{H}=\left\{\varphi: \omega_{\varphi}=\omega+\sqrt{-1} \partial \bar{\partial} \varphi>0, \text { on } M\right\} .
$$

E. Calabi proposed to study the existence of extremal Kähler metric in 1982. This has been a central problem in Kähler geometry since its inception. A Kähler metric is called "extremal" if the complex gradient vector field of its scalar curvature function is holomorphic. A special case is the so-called $c s c K$ metric (constant scalar curvature Kähler metric) when this vector field vanishes. To attack the existence problem of cscK metrics, we propose to study the following continuous path.

For any positive, closed $(1,1)$-form $\chi$, define a continuous path $t \in[0,1]$ as

$$
t \cdot\left(R_{\varphi_{t}}-\frac{\left[C_{1}(M)\right] \cdot[\omega]^{[n-1]}}{[\omega]^{[n]}}\right)=(1-t) \cdot\left(\operatorname{tr}_{\varphi_{t}} \chi-\frac{[\chi] \cdot[\omega]^{[n-1]}}{[\omega]^{[n]}}\right) .
$$

and for simplicity denote

$$
\underline{\chi}=\frac{[\chi] \cdot[\omega]^{[n-1]}}{[\omega]^{[n]}} \quad \text { and } \quad \underline{R}=\frac{\left[C_{1}(M)\right] \cdot[\omega]^{[n-1]}}{[\omega]^{[n]}} .
$$

where $[\omega]^{[k]}=\frac{[\omega]^{k}}{k !}$. Let $C_{t}=(1-t) \underline{\chi}-t \underline{R}$, then Equation (1.1) is rewritten as

$$
(1-t) \operatorname{tr}_{\varphi_{\mathrm{t}}} \chi-\mathrm{t} \mathrm{R}_{\varphi_{\mathrm{t}}}=\mathrm{C}_{\mathrm{t}} .
$$

Definition 1.4 A Kähler metric is called twisted cscK metric if its scalar curvature satisfies Equation (1.2). We call it twisted extremal Kähler metric if the left hand side of Equation (1.2) gives rise to a holomorphic vector field.

When $t=1$, this reduces to the equation for $\csc$ metrics. Let $I$ denote the set of time parameter $t \in[0,1]$ such that Equation (1.1) can be solved at time $t$. As usual, our goal is to first prove that $I$ is not empty which usually means finding a starting point where we can solve this equation. Then, to prove $I$ is open which is crucial for this program to be viable. The hard part is of course to prove $I$ is 
closed which involves hard a priori estimate.

For each $t \in[0,1]$, we call a solution to Equation (1.1) as twisted cscK metric with $(1-t) \chi$. When $t=\frac{1}{2}$, this is exactly the equation considered by Stoppa [77, which in turns was motivated from J. Fine's work [56]. The first theorem we proved here is

Theorem 1.5 (Openness) For any $\chi>0$ and $t \in(0,1)$, if there exists one solution to Equation (1.1) for time $t \in(0,1)$, then there exists a small $\delta>0$ such that for any $t^{\prime} \in(t-\delta, t+\delta) \cap[0,1)$, there exists a solution to Equation (1.1) for time $t^{\prime}$.

This is a crucial result which establishes the validity of the path to solve the cscK metric problem in a given Kähler class. At technical level, it should be compared with the well-known deformation theorem proved by LeBrun-Simanca 62] where they discussed the deformation of cscK metrics when the Kähler class and/or complex structure changes. With the openness of $I$ at present, the next important matter is wether $I$ is non-empty. In particular, we need a starting point.

When $t=0$, this reduces to the well-known equation

$$
\operatorname{tr}_{\varphi} \chi=\underline{\chi}
$$

which is the Euler-Lagrange equation for the well-known $J$ functional introduced in [19], defined by the following formula of its derivative:

$$
\frac{\mathrm{d} J_{\chi}}{\mathrm{d} t}=\int_{M} \frac{\partial \varphi}{\partial t}\left(\operatorname{tr}_{\varphi} \chi-\underline{\chi}\right) \omega_{\varphi}^{[\mathrm{n}]} .
$$

We will delay discussions of $J$ functional to the next section and for now, we just remark that there are several known obstructions to the solution of Equation (1.3). For instance,

$$
\underline{\chi} \cdot[\omega]>[\chi] .
$$

The main point is, if our goal is to attack existence of cscK metrics, we only need to choose one $\chi$ for which $I$ is non-empty. For a moment of thought, it is obvious that we can always solve Equation (1.4) at $t=0$ if we choose $\chi$ to be in the same class as $[\omega]$. Thus, we can always find a smooth positive $(1,1)$-form $\chi$ such that set $I$ is non-empty. In light of the openness theorem 1.5, this leaves the next problem both interesting and important:

Question 1.6 For any $\chi>0$, if there exists one solution to Equation (1.1) for time $t=0$, then there exists a small $\delta>0$ such that for any $t \in[0, \delta) \cap[0,1)$, there exists a solution to Equation (1.1) for time $t$.

We can prove directly that the linearized operator is strictly elliptic or semi-elliptic for $t \in[0,1]$, as we will explain later. The case at $t=0,1$ are subtle for different reason. At time $t=1$, the equation is degenerated elliptic where the kernal is induced by the underlying holomorphic vector field. We encourage readers to 39 for the discussions of this problem. Likewise, we also defer the discussion of the $t=0$ to a later paper. The difficulty cause at $t=0$ is of different nature: while all strictly elliptic operators, the inverse operator lost two derivatives when compairing to $t>0$. Thus, we need to address this problem more carefully. The immediate thought would be use either a version of Nash-Moser inverse function theorem or the method via adiabatic limit as in a beautiful paper [56]. However, there is another different but plausible approach and we would like describe it now. Heuristically, one can take a flow approach as in [28]. One note that at $t=0$, the $J$ flow converges to the solution $\operatorname{tr}_{\varphi} \chi=$ const. In other 
words, for a sufficient small $C^{4, \alpha}$ neighborhood of this solution, the $J$ flow is stable (in other words, for any flow initiate inside this neighborhood will never leave this neighborhood). Intuitively then, the twisted Calabi flow for $t$ small enough is stable in this neighborhood and the argument in 28] might be adopted to settle this problem.

Another interesting twist is to compare this path with Donaldson's Continuity Path on conical KE metrics (see discussions in Section 22). The corresponding problem there is to show the existence of conical Kähler-Einstein metrics when conical angle is very small 1 Note that in the case of conical KE path, the existence for small angle is established through difficult work by R. Berman [6] and JMR [60] etc. It will be interesting to see if the method in [6] can be extend over to our situation. Suppose that there is a sequence of smooth, positive $(1,1)$ form $\chi_{\epsilon}$ such that $\lim _{\epsilon \rightarrow 0} \chi_{\epsilon}=D$ and the convergence is smooth away from divisor $D$, then twisted conical cscK metric path 2.16 and the aformentioned path is naturally connected thorough this limiting process. It will be interesting to see if one can prove from the existence of equation for $t$ small to the existence of conial $\mathrm{KE}$ with small angle, or vice versa. As a matter of fact, we may generalize a conjecture of Donaldson to include the case of twisted cscK metric:

Conjecture 1.7 Suppose $[D]=[\chi]$ and $D$ is generic, then the existence of twisted cscK metric at $t \in[0,1]$ is equivalent to the existence of conical cscK metric at time $t \in[0,1]$.

Note that in canonical class, the necessary part is established in [25] and the sufficient part is open. If we drop the assumption that $D$ is generic, then one expect the sufficient part to be false. It is interesting if we can re-construct Tian-Yau's theorem on complete Calabi-Yau metric on Kähler manifold outside a divisor or the existence of conical KE metric with small conical angle via twisted cscK metric approached suggested here. While technically it is still complicated, the conceptual picture is nonetheless very clear.

One surprising observation is that we can avoid this problem of "jump" or regularity at $t=0$ in Fano manifold by working on a slightly different path (2.12). 2 Note that in Fano manifold, in any Kähler class, we can find a metric $\omega$ such that

$$
\text { Ric } \omega>0 \text {. }
$$

If we choose $\chi=$ Ric $\omega>0$, then

Corollary 1.8 (Openness) Suppose $\chi$ is a positive Ricci form in any Fano manifold. Then for any $t \in[0,1)$, if there exists one solution to Equation (1.1) for time $t \in[0,1)$, then there exists a small $\delta>0$ such that for any $t^{\prime} \in[t-\delta, t+\delta) \cap[0,1)$, there exists a solution to Equation (2.12) for time $t^{\prime}$.

Let $E$ be the well-known K-energy functional introduced by T. Mabuchi [66, whose derivative is given below:

we then call

$$
\frac{\mathrm{d} E}{\mathrm{~d} t}=-\int_{M} \frac{\partial \varphi}{\partial t}\left(R_{\varphi}-\underline{R}\right) \omega_{\varphi}^{[n]}
$$

$$
E_{\chi, t}=(1-t) J_{\chi}+t E
$$

the twisted K-energy functional.

Following Stoppa [77] and Donaldson [47, we have

Proposition 1.9 For any $\chi$ and $t \in[0,1], \omega \rightarrow(1-t) \operatorname{tr}_{\varphi_{\mathrm{t}}} \chi-\mathrm{tR}_{\varphi_{\mathrm{t}}}-\mathrm{C}_{\mathrm{t}}$ can be viewed as a moment map associated with the infinite dimensional group of exact symplectic diffeomorphisms.

\footnotetext{
${ }^{1}$ Suppose that $[D]=[\chi]=C_{1}(M)$, then the path we consider can be reduced to the usual Donaldson's path on conical KE metrics.

${ }^{2}$ The path (2.12) at $[0,1]$ is equivalent to the path (1.1) at $\left[\frac{1}{2}, 1\right]$.
} 
Following Donaldson [47, Stoppa [77] and in particular, Lejmi-Székelyhidi 64, we can also formulate a notion of twisted K-stability which we will delay the discussions to Section [5. In [18, we proved that $J$ functional is strictly convex along $C^{1,1}$ geodesic segments. Following the recent work of BermanBerndtsson [7, Chen-Li-Paun [16], we arrive at the following expected statement

Proposition 1.10 For any $t \in[0,1]$, the twisted functional $E_{\chi, t}$ is convex along any $C^{1,1}$ geodesic segment and in particular, strictly convex when $t<1$.

When $t=0$, this is due to [18; when $t=1$, this was first conjectured by the author and proved in recent works [7, 16. The proposition above is essentially a combination of these two results (cf. [7, 16]) . As corollary, we proved that

Corollary 1.11 For any $t \in[0,1]$, the functional $E_{\chi, t}$ is bounded from below if Equation (1.1) has a solution for $t$.

Corollary 1.12 For any $t \in[0,1)$, the twisted cscK metric is unique in its Kähler class.

We can certainly reformulate the well-known YTD conjecture for the twisted cscK metric.

Conjecture 1.13 There exists a twisted cscK metric if and only if it is twisted K-stable.

We also believe that we should have a corresponding notion of twisted Paul's stability which is equivalent to the existence of twisted cscK metric. It is well known that the existence of Kähler-Einstein metrics implies also Paul's stability if $\operatorname{Aut}(M, J)$ is discrete. Conversely, Paul's stability implies that the K-energy is proper in all finite Bergman spaces [68] . For semi-stable orbits, we believe the following conjecture holds

Conjecture 1.14 If $(M,[\omega], J)$ is destabilized by $\left(M,[\omega], J^{\prime}\right)$ (where the second one admits a cscK metric). Then for any $\chi \in[\omega]$, there exists a unique twisted cscK metric for $(1-s) R_{\varphi}-s \operatorname{tr}_{\varphi} \chi=\mathrm{c}_{\mathrm{s}}$.

Theorem 1.15 ([39]) Let $\chi>0$ be any closed, positive $(1,1)$-form in [ $\omega]$. If there exists a cscK metric, then for $t \in[0,1]$ close enough to 1, there exists a solution to Equation (1.1).

We really follow the idea of Bando-Mabuchi to solve the path for $1-t$ small enough. In their case, they need to solve the Aubin continuity path along the way from $t=1$ to $t=0$. In the present situation, it is technically not feasible to do "weak compactness" for a family of twisted cscK metrics yet. Nonethelese, the problem of weak compactness of twisted cscK metric is one of the fundamental problems one ultimately need to address (cf. [83] 38] and references therein for discussion on this important topics).

\subsection{Related problems}

Following our discussions about this one parameter path (Equation (1.1)), it makes sense to define

Definition 1.16 For any $\chi>0$, define $R(\chi)$ to be the supremum of time $t \in[0,1]$ for which this equation is solvable.

In view of the openness theorem(Theorem 1.8), we make the following conjecture:

Conjecture 1.17 For any two closed, positive (1,1)-forms $\chi_{1}, \chi_{2}$ in the same cohomology class, we have

$$
R\left(\chi_{1}\right)=R\left(\chi_{2}\right) .
$$


In the classical Aubin's continuous path, this conjecture was proved by G. Székelyhidi 79. More interestingly, together with T. Collins in [41] they also proved that if this path is solvable for $\chi_{1}>0$ at time $t=0$, then it is also solvable for $\chi_{2}>0$ at $t=0$ provided that $\left[\chi_{1}\right]=\left[\chi_{2}\right]$. It will be really interesting to answer

Question 1.18 Can we characterize the polarized Kähler manifold $(M,[\omega])$ where there exists a $(1,1)$ form $\chi>0$ such that $R(\chi)=1$ but with no cscK metrics in $(M,[\omega])$ ?

Another important problem is to study the following twisted Calabi flow for any $t \in[0,1]$, where the family of Kähler potentials $\varphi=\varphi_{s}$ evolves by:

$$
\frac{\partial \varphi}{\partial s}=t R_{\varphi}-(1-t) \operatorname{tr}_{\varphi} \chi+\mathrm{C}_{\mathrm{t}} .
$$

It is interesting and important to establish the short time existence for proper initial metrics. The author believes this is true, along with a stability theorem at the neighborhood of twisted cscK metric (as in 28 for the usual Calabi flow). Note that in Riemann surface [61, J. Pook proved short and long time existence of the twisted Calabi flow where the form depends on time $t>0$. More importantly, one can adopt two important conjectures about the Calabi flow to the twisted case here.

Conjecture 1.19 (Chen) The twisted Calabi flow exists globally for any smooth initial Kähler potential.

Similarly, we can re-formulate Donaldson's conjectural pictures at the case of Calabi flow to our settings:

Conjecture 1.20 Suppose the twisted Calabi flows have global existence. Then the asymptotic behavior of the twisted Calabi flow starting from $(M, \omega, \chi, J)$ falls into the following possibilities:

1. The flow converges to a twisted cscK metric on the same complex manifold $(M, J)$;

2. The flow converges, up to a differmorphism, to a twisted extremal Kähler metric;

3. The manifold does not admit a twisted cscK metric or twisted extremal metric but the transformed flow $\left(M,\left[\omega_{t}\right], \chi_{t}, J_{t}\right)$ converges to $\left(Y,\left[\omega_{\infty}\right], \chi^{\prime}, J^{\prime}\right)$ which forms a twisted extremal Kähler metric, possibly with at least codimension 2 singularities.

More interestingly, it will be nice to understand the important result of J. Streets [78] as well as more recent exciting result on the Calabi flow [65] in the twisted Calabi flow case. We believe the result can be made more precise because of the strict convexity of the twisted K-energy functional.

Another important conjecture we want to raise is

Conjecture 1.21 For any $t \in(0,1)$, the twisted functional $E_{\chi, t}$ is proper in terms of geodesic distance function if and only if Equation (1.1) has a solution.

Note that when $t=1$, this is the conjecture on the properness of the K-energy functional ( see Conjecture 3.3 below) . We remark that this is another reason why we believe Conjecture 1.17 is correct. In Section 3 we will prove the necessary part of this conjecture.

Acknowledgement The author wishes to thank his students Yu Zeng and Chengjian Yao for critical help through the preparation of this paper. Very recently, Yoshinori Hashimoto informed us that he can solve question 1.6 with an outline of proof. His proof can also be extended to the case $t \in(0,1)$. 


\section{Discussions about various known paths}

According to E. Calabi, the famous Kähler-Einstein problem can be reduced to a problem of complex Monge-Ampère equation:

$$
\operatorname{det}\left(g_{\alpha \bar{\beta}}+\frac{\partial^{2} \varphi}{\partial z_{\alpha} \partial \bar{z}_{\beta}}\right)=e^{-\varphi+h_{\omega}} \operatorname{det}\left(g_{\alpha \bar{\beta}}\right)
$$

where

$$
\omega_{g}+\sqrt{-1} \partial \bar{\partial} \varphi>0 \text { on } \mathrm{M}
$$

To attack the existence problem, one adopts a continuous path

$$
\operatorname{det}\left(g_{\alpha \bar{\beta}}+\frac{\partial^{2} \varphi}{\partial z_{\alpha} \partial \bar{z}_{\beta}}\right)=e^{-t \varphi+h_{\omega}} \operatorname{det}\left(g_{\alpha \bar{\beta}}\right), \quad t \in[0,1] .
$$

When $t=0$, this is Calabi's volume conjecture. The strategy is to prove the set

$$
\{t: \in[0,1] \mid \text { Equation (2.7) can be solved at time } t\}
$$

is both open and closed. The openness is more or less standard. To derive closedness, we need to obtain a priori estimate which is independent of $t$. The memorial feature of the resolution of the Calabi conjecture by S.-T. Yau is to reduce the second order estimate to a $C^{0}$ estimate on Kähler potential, which is followed by a $C^{3}$ estimate of E. Calabi. This is of course well-known to Kähler geometers. However, the following might be less well-known. A cscK metric equation can be decomposed as a coupled second order equations which we will describe below.

$$
\begin{aligned}
\log \frac{\operatorname{det}\left(g_{\alpha \bar{\beta}}+\frac{\partial^{2} \varphi}{\partial z_{\alpha} \partial \bar{z}_{\beta}}\right)}{\operatorname{det}\left(g_{\alpha \bar{\beta}}\right)} & =F, \\
\Delta_{\varphi} F & =-\underline{R}+\operatorname{tr}_{\varphi} \operatorname{Ric}_{\mathrm{g}}
\end{aligned}
$$

The following proposition is known:

Proposition 2.1 If $g_{\varphi}$ is a cscK metric and it is quasi isometric to the background metric $g$, then the $C^{4, \alpha}$ norm on $\varphi$ is uniformly controlled.

Here is a few words about the proof of this proposition: since $g_{\varphi}$ is quasi-isometric, then Equation (2.9) is uniformly elliptic with a bounded right hand side. Therefore, by De Giorgi, $[F]_{C^{\alpha}(M, g)}$ is uniformly bounded. Substituting this into Equation (2.8), it becomes a complex Monge-Ampère equation with $C^{\alpha}$ bound on the right hand side. According to theory of Caffarelli, Evans-Krylov and the recent observation of Y. Wang [91] (cf. also Chen-Wang [37), we know $[\varphi]_{C^{2, \alpha}(M, g)}$ is uniformly controlled. Iterating from Equation (2.8) and (2.9) once more, we get $[\varphi]_{C^{4, \alpha}(M, g)}$ is uniformly controlled.

Inspired by this proposition, we propose the following naively looking equation:

$$
\begin{aligned}
\log \frac{\operatorname{det}\left(g_{\alpha \bar{\beta}}+\frac{\partial^{2} \varphi}{\partial z_{\alpha} \partial z_{\beta}}\right)}{\operatorname{det}\left(g_{\alpha \bar{\beta}}\right)} & =F \\
\Delta_{\varphi} F & =t\left(-\underline{R}+\operatorname{tr}_{\varphi} \text { Ric g }\right)
\end{aligned}
$$

for any $t \in[0,1]$. Set $I$ to be all $t \in[0,1]$ such that the above equation can be solved. Then, obviously $0 \in I$. since it reduces to the well-known Calabi's volume conjecture albeit it is trivial in this setting. 
The fundamental problem is if the set $I$ necessarily open? It is hard to tell directly the answer to this crucial question. More importantly, it is quite difficult to imagine the role of the sign of "Ric $g$ " played in this family of equations. Purely from PDE consideration, I think both positivity and negativity of "Ric $g$ " have their own advantages and pitfalls. However, these two signs are distinctly different from geometric perspective. In fact, the openness depends crucially on the sign of "Ric $g$ ". It is open if it is positive. This can be readily seen if we re-write this path of pair of equations as

$$
R_{\varphi}=(1-t) \operatorname{tr}_{\varphi} \operatorname{Ric} \mathrm{g}+\mathrm{t} \underline{\mathrm{R}}
$$

This is very similar to Equation (1.1) with $\chi=$ Ric $g$. Thus, in light of Theorem 1.8 the openness holds as long as Ric $g>0$. It is not clear what happens if Ric $g<0$.

On the other hand, we observed that there is an untraced version of this continuous path, which is the well-known Aubin path. Note that in Kähler-Einstein settings, the Aubin path reads as

$$
(1-t) \chi-t \operatorname{Ric} \omega=\frac{C_{t}}{n} \omega
$$

This casts the "traditional Aubin path (2.7)" into new light: that this is also a continuous family of twisted $\operatorname{cscK}$ metrics over parameter $t \in[0,1]$. It is not difficult to see

Proposition 2.2 Along the Aubin path, if $(M,[\omega])$ is $K$ semi-stable, then for every $t \in[0,1)$, it is twisted K-stable.

Following strategy of [25, 26, 27, we may ask

Question 2.3 If there exists a sequence of twisted cscK metric $\omega_{i}$ for $\left(M,[\omega], \chi, t_{i}\right)$ where $t_{i} \rightarrow \bar{t}$ such that

1. $(M,[\omega], \chi, \bar{t})$ is twisted $K$-stable;

2. Partial $C^{0}$ estimates holds for $\left(M, \omega_{i}, \chi, t_{i}\right)$, then does $(M,[\omega], \chi, \bar{t})$ admit twisted cscK metric?

According to G. Szekelyhid [80, who in turns follows [26], the partial $C^{0}$ estimate holds for a sequence of Kähler metrics over Aubin path. If the answer to aformentioned question is true, then we can prove Yau's stability conjecture by following Aubin's path. This leaves a very interesting question

Question 2.4 For any sequence of twisted cscK metric $\omega_{i}$ for $\left(M,[\omega], \chi_{i}, t_{i}\right)$, when does the partial $C^{0}$ estimate hold?

In the singular case where $\chi=2 \pi[D]$ for a divisor $D \subset M$, this equation reduces to

$$
\text { Ric } \omega=-\frac{C_{t}}{n t} \omega+2 \pi \frac{1-t}{t}[D]
$$

it provides a variant of Donaldson's continuity path of conical Kähler-Einstein metrics [52, Equation (27)]. The question is wether the parallel theory holds in this case?

This continuity path could be viewed as a natural generalization of the classical Aubin's continuity path

$$
\text { Ric } \omega_{t}=t \omega+(1-t) \chi
$$

concerning the study of Kähler-Einstein metrics. Taking trace of the equation (2.14) gives the notion of "conical cscK metric":

$$
R_{\varphi}=-\frac{C_{t}}{t}+2 \pi \frac{1-t}{t} \operatorname{tr}_{\varphi}[\mathrm{D}]
$$

We call $\omega$ this conical constant scalar curvature Kähler metric if it satisfies: 
1. It is quasi isometric to a Kähler metric with cone angle $2 \pi t$ near the divisor $D$;

2. The metric tensor is in $C^{\alpha, t}$ near divisor $D$ for some $\alpha \in\left(0, \min \left(1, \frac{1}{t}-1\right)\right)$;

3. The scalar curvature is constant outside the divisor $D$.

We could ask the following

Question 2.5 If there is no tangential holomorphic vector in $(M, D)$, can we deform the conical cscK metric with angle $t>0$ ? More interestingly, do we always have a conical cscK metric for $t$ small enough?

\section{Convexity and moment map pictures}

\subsection{K-energy functional and moment map picture}

One memorial feature is that, via the work of S. Donaldson [46] and Fujiki, the scalar curvature (regarded as the Lie algebra element) of a Kähler metric can be viewed as a moment map for an action of the group of exact sympletic diffeomorphisms on the space of all compatible almost complex structures. In 1998, the author [17] established the existence of $C^{1,1}$ geodesic segment between two smooth Kähler potentials and proved that the $\mathrm{K}$-energy functional is convex along $C^{1,1}$ geodesic segment if $C_{1} \leq 0$. In general, the best regularity for geodesic segment might be $C^{1,1}$ only. At the time, the best we can prove is the following

Theorem $3.1\left([21,[30])\right.$ Suppose $\varphi_{0}, \varphi_{1}$ are two Kähler potential and $\varphi_{t}(t \in[0,1])$ is the $C^{1,1}$ geodesic segment connecting $\varphi_{0}$ to $\varphi_{1}$. Then,

$$
\left.\left(\mathrm{d} E,\left.\varphi_{t}^{\prime}\right|_{t=0}\right)\right|_{\varphi_{0}} \leq\left.\left(\mathrm{d} E,\left.\varphi_{t}^{\prime}\right|_{t=1}\right)\right|_{\varphi_{1}}
$$

However, this theorem leads to the following conjecture [19] by the author.

Conjecture 3.2 The K-energy functional is convex on any $C^{1,1}$ geodesic segment.

New understanding in Kähler geometry leads to a proof to this full conjecture by the work of BermanBerndtsson [7] and independently by Chen-Li-Paun [16]. Another related conjecture is the following

Conjecture 3.3 There exists a cscK metric if and only if the K-energy functional is coercive in terms of geodesic distance to the maximal invariant, totally geodesic subspace induced by automorphism group.

This should be seen as a continuation of Conjecture 1.21. In Kähler-Einstein manifold without holomophic vector field, Ding-Tian proved that the K-energy functional is proper in terms of Aubin functional. The corresponding conjecture (in terms of geodesic distance) has very little progress. Very recenty, in Fano manifold with no holomorphic vector field, T. Darvas 42 proved that the Ding functional is proper in terms of $L^{1}$ geodesic distance. This work gives a very good indication that the full conjecture above should be true (module some mild modifications). In toric surface and for $t=1, \mathrm{X}$.-H Zhu and B. Zhou can establish a weak existence of extremal Kähler metric if the modified K-energy functional is proper 95. This conjecture more likely can be established in toric surface settings first.

Now we give a proof of the necessary part of Conjecture 1.21 with the assumption that $J_{\chi}$ is bounded from below. 
Proof The following functional is well-known

$$
J(\varphi)=\int_{M} \varphi\left(\omega^{n}-\omega_{\varphi}^{n}\right)=\int_{M} \sqrt{-1} \partial \varphi \wedge \bar{\partial} \varphi \wedge\left(\sum_{k=0}^{n-1} \omega^{k} \wedge \omega_{\varphi}^{n-k-1}\right)>0 .
$$

According to T. Davas's recent work [42, 43, this functional is equivalent to geodesic distance in $\mathcal{H}$ with respect to Mabuchi's metric. Recall a decomposition formula of K-energy in [19]: For any $\phi \in \mathcal{H}_{\omega}$, we have

$$
E(\varphi)=\int_{M} \ln \frac{\omega_{\varphi}^{n}}{\omega_{0}^{n}} \omega^{n}-J_{R i c} \omega_{0}(\varphi) .
$$

where $J_{R i c \omega_{0}}$ is the $J_{\chi}$ functional defined by Formula 1.4 for $\chi=R i c \omega_{0}$.

The first term of this decomposition formula (of K-energy) is called Entropy functional and we will denote it as $E_{0}(\varphi)$. We claim that Entropy functional is proper in terms of geodesic distance. According to Davas's recent work [42, 43, we only need to prove the entropy functionl dominates this $J$ functional. This is a well-known fact and we include a short proof here for completeness (cf. 21] for this calculation). According G. Tian, there is a positive constant $\alpha>0$ which depends only on the Kähler class $[\omega]$ such that for any $\varphi \in \mathcal{H}$, we have

$$
\int_{M} e^{-\alpha(\varphi-\sup \varphi)} \omega^{n} \leq C,
$$

or

$$
\int_{M} e^{-\alpha(\varphi-\sup \varphi)-\log \frac{\omega_{\varphi}^{n}}{\omega^{n}}} \omega_{\varphi}^{n} \leq C
$$

If we set

$$
\int_{M} \omega^{n}=1
$$

we have

$$
\int_{M}-\alpha(\varphi-\sup \varphi)-\log \frac{\omega_{\varphi}^{n}}{\omega^{n}} \omega_{\varphi}^{n} \leq C
$$

Therefore,

$$
\begin{aligned}
\alpha \sup \varphi & \leq \alpha \int_{M} \varphi \omega_{\varphi}^{n}+\int_{M} \log \frac{\omega_{\varphi}^{n}}{\omega_{\varphi}^{n}} \omega_{\varphi}^{n} \\
& \leq \alpha \int_{M} \varphi \omega_{\varphi}^{n}+\int_{M} \log \frac{\omega_{\varphi}^{n}}{\omega^{n}} \omega_{\varphi}^{n} .
\end{aligned}
$$

In other words,

$$
\alpha \int_{M} \varphi \omega^{n} \leq \alpha \sup \varphi \leq \alpha \int_{M} \varphi \omega_{\varphi}^{n}+\int_{M} \log \frac{\omega_{\varphi}^{n}}{\omega^{n}} \omega_{\varphi}^{n}
$$

Consequently,

$$
\alpha J(\varphi)=\alpha \int_{M} \varphi\left(\omega^{n}-\omega_{\varphi}^{n}\right) \leq \int_{M} \log \frac{\omega_{\varphi}^{n}}{\omega^{n}} \omega_{\varphi}^{n} .
$$

Thus, the entropy functional is proper in terms of geodesic distance.

Now, we are ready to prove the necessary part of this theorem. We assume that

$$
\operatorname{tr}_{\varphi} \chi=\frac{[\chi] \cdot[\omega]^{[\mathrm{n}-1]}}{[\omega]^{[\mathrm{n}]}}
$$

can be solved in $[\omega]$. By convexity, we know that $J_{\chi}$ has lower bound in $\mathcal{H}$ first. Then, for $\epsilon>0$ small enough, we have

$$
J_{\chi} \geq \pm \epsilon J_{R i c \omega}-C .
$$

This is because we can solve the corresponding equation

$$
\operatorname{tr}_{\varphi}(\chi \pm \epsilon \operatorname{Ric} \omega)=\frac{[\chi] \cdot[\omega]^{[\mathrm{n}-1]}}{[\omega]^{[\mathrm{n}]}}+\epsilon \frac{\left[\mathrm{C}_{1}(\mathrm{M})\right] \cdot[\omega]^{[\mathrm{n}-1]}}{[\omega]^{[\mathrm{n}]}}
$$


via perturbation for small enough $\epsilon>0$. The desired inequality then follows from convexity of $J_{\chi \pm \epsilon R i c \omega}$ functional over $C^{1,1}$ geodesic segment in $\mathcal{H}$.

Suppose $\omega_{\varphi}$ is a twisted cscK metric for $\chi>0$ and $t_{0} \in(0,1)$, then there exists a small $\delta>0$ such that the twisted $\csc K$ metric equation can be solved for any $t^{\prime} \in\left(t_{0}, t_{0}+2 \delta\right) \subset(0,1)$. Fix $t^{\prime}=t_{0}+\delta$. Then,

$$
E+\frac{1-t^{\prime}}{t^{\prime}} J_{\chi} \geq-C
$$

Note that the coefficient $\frac{1-t^{\prime}}{t^{\prime}}$ is strictly less than $\frac{1-t_{0}}{t_{0}}$. In other words, this inequality holds for any number close enough to $\frac{1-t_{0}}{t_{0}}$. Now

$$
\begin{aligned}
t_{0} E+\left(1-t_{0}\right) J_{\chi} & =\left(t_{0}-\epsilon\right) E+\left(1-t_{0}-\delta^{\prime}\right) J_{\chi}+\epsilon E+\delta^{\prime} J_{\chi} \\
& =\left(t_{0}-\epsilon\right)\left(E+\frac{1-t_{0}-\delta^{\prime}}{t_{0}-\epsilon} J_{\chi}\right)+\epsilon E_{0}+\left(\delta^{\prime} J_{\chi}+\epsilon J_{R i c} \omega\right)+\epsilon \underline{R} I(\varphi) .
\end{aligned}
$$

Now, for fixed $\delta^{\prime}$ small enough, we can find $\epsilon$ small enough so that

$$
\delta^{\prime} J_{\chi}+\epsilon J_{R i c} \omega \geq-C
$$

and

$$
E+\frac{1-t_{0}-\delta^{\prime}}{t_{0}-\epsilon} J_{\chi} \geq-C
$$

Thus, we prove

$$
t_{0} E+\left(1-t_{0}\right) J_{\chi} \geq \epsilon E_{0}-C
$$

It follows that the twisted K-energy is proper in terms of geodesic distance.

In summary, we prove

Theorem 3.4 Suppose that

$$
\operatorname{tr}_{\varphi} \chi=\frac{[\chi] \cdot[\omega]^{[\mathrm{n}-1]}}{[\omega]^{[\mathrm{n}]}}
$$

can be solved in $[\omega]$. For any $t \in(0,1)$, the twisted K-energy functional is coercive in terms of geodesic distance if one of the following condition holds:

1. There exists a constant scalar curvature metric;

2. The K-energy functional is bounded from below;

3. There existed a twisted cscK metric for $t \in(0,1)$.

Conjecture 3.5 For any $C^{1,1}$ Kähler potential, we can have a Calabi flow or twisted Calabi flow initiated from this potential and the flow becomes instantly smooth. Consequently, the $C^{1,1}$ minimizer of the twisted K-energy is always smooth.

In canonical Kähler class, both statements has been asserted true by the efforts of many mathematicians. The weak Kähler Ricci flow was initially introduced to obtain partial regularity for $C^{1,1}$ minimizer on the K-energy functional in [20] and subsequently [23] and [24]. Now, the weak Ricci flow is itself an intensive subject of study: there are lots of interesting results on the regularity properties starting from weak data, see [53, 54, 59, 45, 67, for a partial list and reference therein. 


\section{$3.2 \quad J$ functional}

In [18, the author introduce the so-called $J$-flow as

$$
\frac{\partial \varphi}{\partial t}=\underline{\chi}-\operatorname{tr}_{\varphi} \chi
$$

This is used to study the lower bound of the K-energy functional on ample Kähler manifold. A striking feature of this $J$ flow is its convexity along any $C^{1,1}$ geodesic segment.

Proposition 3.6 ([18]) $J$ is a strictly convex functional along any $C^{1,1}$ geodesic. In particular, $J$ has at most one critical point in $\mathcal{H}$.

For the convenience of readers, we re-produce the proof here.

Proof Suppose $\varphi_{t}$ is a $C^{1.1}$ geodesic. In other words, $\varphi_{t}$ is a weak limit of the following continuous equation as $\epsilon \rightarrow 0$ (with uniform bounds on the second mixed derivatives of Kähler potentials):

$$
\left(\frac{\partial^{2} \varphi}{\partial t^{2}}-\frac{1}{2}\left|\nabla \frac{\partial \varphi}{\partial t}\right|_{\varphi}^{2}\right) \frac{\omega_{\varphi_{t}}^{n}}{n !}=\epsilon \cdot \frac{\omega_{0}^{n}}{n !}
$$

Denote $g_{t}$ as the corresponding Kähler metric corresponds to the Kähler form $\omega_{\varphi_{t}}$. Again, we drop the dependence of $t$ from $g_{t}$ for convenience from now on. Recall the definition of $J$, we have

$$
\frac{\mathrm{d} J_{\chi}}{d t}=\int_{V} \frac{\partial \varphi}{\partial t}\left(g^{\alpha \bar{\beta}} \chi_{\alpha \bar{\beta}}\right) \frac{\omega_{\varphi}^{n}}{n !} .
$$

Then (denote $\sigma=g^{\alpha \bar{\beta}} \chi_{\alpha \bar{\beta}}$ in the following calculation):

$$
\begin{aligned}
\frac{d^{2} J}{d t^{2}}= & \int_{V}\left(\frac{\partial^{2} \varphi}{\partial t^{2}} \sigma-\frac{\partial \varphi}{\partial t} g^{\alpha \bar{\beta}}\left(\frac{\partial \varphi}{\partial t}\right)_{, \bar{\beta} r} g^{r \bar{\delta}} \chi_{\alpha \bar{\delta}}+\frac{\partial \varphi}{\partial t} \sigma \Delta_{g} \frac{\partial \varphi}{\partial t}\right) \frac{\omega_{\varphi}^{n}}{n !} \\
= & \int_{V}\left(\frac{\partial^{2} \varphi}{\partial t^{2}} \sigma-\frac{\partial \varphi}{\partial t} g^{\alpha \bar{\beta}}\left(\frac{\partial \varphi}{\partial t}\right)_{, \bar{\beta} r} g^{r \bar{\delta}} \chi_{\alpha \bar{\delta}}\right. \\
= & \left.\int_{V}\left(\left(\frac{\partial^{2} \varphi}{\partial t^{2}}-\frac{1}{2} \mid \nabla \frac{\partial \varphi}{\partial t}\right)_{, r} \sigma g_{g}^{r}\right) \sigma-\frac{\partial \varphi}{\partial t} g^{\alpha \bar{\beta}}\left(\frac{\partial \varphi}{\partial t}\right)_{, \bar{\beta} r} g^{r \bar{\delta}} \chi_{\alpha \bar{\delta}}-\frac{\partial \varphi}{\partial t} g^{\alpha \bar{\beta}} \chi_{\alpha \bar{\beta}, \bar{g}} g^{\bar{\delta}}\left(\frac{\partial \varphi}{\partial t}\right)_{, r}\right) \frac{\omega_{\varphi}^{n}}{n !} \\
= & \left.\quad-\frac{\partial \varphi}{\partial t}\left(g^{\alpha \bar{\beta}} \chi_{\alpha \bar{\delta}} g^{r \bar{\delta}}\right)_{\overline{,}}\left(\frac{\partial \varphi}{\partial t}\right)_{, r}\right)_{\frac{\omega_{\varphi}^{n}}{n !}}\left(\left(\frac{\partial^{2} \varphi}{\partial t^{2}}-\frac{1}{2}\left|\nabla \frac{\partial \varphi}{\partial t}\right|_{g}^{2}\right)\left(g^{\alpha \bar{\beta}} \chi_{\alpha \bar{\beta}}\right)+\left(\frac{\partial \varphi}{\partial t}\right)_{, \bar{\beta}}\left(g^{\alpha \bar{\beta}} \chi_{\alpha \bar{\delta}} g^{r \bar{\delta}}\right)\left(\frac{\partial \varphi}{\partial t}\right)_{, r}\right) \frac{\omega_{\varphi}^{n}}{n !} \\
\geq & \int_{V}\left(\frac{\partial \varphi}{\partial t}\right)_{, \bar{\beta}}\left(g^{\alpha \bar{\beta}} \chi_{\alpha \bar{\delta}} g^{r \bar{\delta}}\right)\left(\frac{\partial \varphi}{\partial t}\right)_{, r} \frac{\omega_{\varphi}^{n}}{n !} \geq 0 .
\end{aligned}
$$

The last equality holds along any $C^{1,1}$ geodesic.

Since its inception, the flow is now well studied. According to Song-Weinkove [75], the necessary and sufficient condition for the flow to converge is that there exists a form $\omega^{\prime} \in[\omega]$ such that

$$
\left(n \underline{\chi} \omega^{\prime}-(n-1) \chi\right) \wedge \omega^{\prime n-2}>0 .
$$

For more updated work on this subject, we refer readers to Weinkove [92, Song-Weinkove [75] and FangLai-Song-Weinkove [55] for further readings.

Following this proposition and the recent works [7, 16, we can easily prove that the twisted Kenergy functional is convex (Propoisition 1.10), and bounded from below if there is a twisted cscK metric (Corollary 1.11) and finally can prove the uniqueness of twisted cscK metric for $t<1$. 


\section{Deformation}

In this section, we will prove the openness for deformation of twisted $\operatorname{cscK}$ metrics (Theorem 1.8). Now we assume $t \in(0,1)$. Set

$$
\mathcal{H}^{4, \alpha}(M)=\left\{\varphi \in C^{4, \alpha}(M, \mathbb{R}) \mid \omega_{\varphi}=\omega+\sqrt{-1} \partial \bar{\partial} \varphi>0\right\}
$$

For any closed positive $(1,1)$-form $\chi$ on $M$, we define a map

$$
\begin{aligned}
F: \mathcal{H}^{4, \alpha}(M) \times[0,1] & \longrightarrow C^{\alpha}(M, \mathbb{R}) \times[0,1] \\
(\varphi, t) & \longmapsto\left(t\left(R_{\varphi}-\underline{R}\right)-(1-t)\left(\operatorname{tr}_{\varphi} \chi-\underline{\chi}\right), \mathrm{t}\right)
\end{aligned}
$$

where $R_{\varphi}$ is the scalar curvature of $\omega_{\varphi}$. The openness theorem $0<t<1$ is equivalent to the following 3 .

Theorem 4.1 If $F\left(\varphi_{0}, t_{0}\right)=\left(0, t_{0}\right)$ for some $t_{0} \in(0,1)$, then for $t \in[0,1)$ which is sufficiently close to $t_{0}$, we can find $\varphi=\varphi(t)$ such that $F(\varphi, t)=(0, t)$.

Consider the linearization of $F$ :

$$
\begin{aligned}
\left.\mathcal{D} F\right|_{(\varphi, t)}: C^{4, \alpha}(M) \times \mathbb{R} & \longrightarrow C^{\alpha}(M) \times \mathbb{R} \\
(u, s) & \longmapsto\left(\mathcal{L}_{(\varphi, t)} u+s\left(R_{\varphi}-\underline{R}+\operatorname{tr}_{\varphi} \chi-\underline{\chi}\right), \mathrm{s}\right)
\end{aligned}
$$

where $\mathcal{L}_{(\varphi, t)}$ is the linearized operator of the twisted scalar curvature function, i.e.

$$
\mathcal{L}_{(\varphi, t)} u=-t \Delta_{\varphi}^{2} u-\left\langle\sqrt{-1} \partial \bar{\partial} u, t \operatorname{Ric}_{\varphi}-(1-t) \chi\right\rangle_{\varphi}
$$

Set $T=\left.\mathcal{D} F\right|_{\left(\varphi_{0}, t_{0}\right)}$, then

Lemma $4.2 \mathcal{R}(T)$, which is the range of $T$, is closed.

Before we prove this lemma, it is important to note that

Lemma 4.3 The kernel of $T$ is one dimensional for any $(\varphi, t)$ which is sufficiently close to $\left(\varphi_{0}, t_{0}\right)$ in $C^{4, \alpha}(M) \times \mathbb{R}$.

Proof For any $(u, s) \in \operatorname{Ker} T$, without loss of generality we might assume that

$$
\int_{M} u \omega_{\varphi}^{n}=0
$$

By definition, we have $s=0$ and

$$
\mathcal{L}_{(\varphi, t)} u=0
$$

Thus,

$$
\begin{aligned}
\int u \mathcal{L}_{(\varphi, t)} u \omega_{\varphi}^{n} & =\int\left(-t \Delta_{\varphi}^{2} u-t u_{, \bar{\alpha} \beta}\left(\operatorname{Ric}_{\varphi}\right)_{\alpha \bar{\beta}}+(1-t) u_{, \bar{\alpha} \beta} \chi_{\alpha \bar{\beta}}\right) u \omega_{\varphi}^{n} \\
& =\int\left(-t\left|u_{, \bar{\alpha} \bar{\beta}}\right|_{\varphi}^{2}-(1-t) u_{, \bar{\alpha}} u_{, \beta} \chi_{\alpha \bar{\beta}}+u_{, \bar{\delta}}\left(t R_{\varphi}-(1-t) \operatorname{tr}_{\varphi} \chi\right)_{, \delta} \mathrm{u}\right) \omega_{\varphi}^{\mathrm{n}}
\end{aligned}
$$

\footnotetext{
${ }^{3}$ We will deal with the case $t=0$ elsewhere.
} 
It follows that

$$
\begin{aligned}
& (1-t) \int u_{, \bar{\alpha}} u_{, \beta} \chi_{\alpha \bar{\beta}} \omega_{\varphi}^{n}=-\int t\left|u_{, \bar{\alpha} \bar{\beta}}\right|_{\varphi}^{2} \omega_{\varphi^{n}}+\int u_{, \bar{\delta}}\left(t R_{\varphi}-(1-t) \operatorname{tr}_{\varphi} \chi\right)_{, \delta} \mathrm{u} \omega_{\varphi}^{\mathrm{n}} \\
& \leq-\int t\left|u_{, \bar{\alpha} \bar{\beta}}\right|_{\varphi}^{2}+\epsilon\left(\int u^{2}+\int|\nabla u|_{\varphi}^{2}\right) \\
& \leq-(1-\epsilon) t \int\left|u_{, \bar{\alpha} \bar{\beta}}\right|_{\varphi}^{2}+\epsilon(C+1) \int|\nabla u|_{\varphi}^{2} \text {. }
\end{aligned}
$$

Here $C$ is the Poincaré constant with respect to the metric $\omega_{\varphi}$ and $\epsilon$ is controlled by the quantity:

$$
\sup _{M}\left|\nabla\left(t R_{\varphi}-(1-t) \operatorname{tr}_{\varphi} \chi\right)\right|
$$

which could be made as small as we want for choosing $(\varphi, t)$ sufficiently close to $\left(\varphi_{0}, t_{0}\right)$. Thus, for any $t_{0}<1$, we can choose a small neighborhood of $t_{0}$ such that

$$
(1-t) c>\epsilon(1+C)
$$

here $2 c$ is the lower bound of $\chi$ in terms of $\omega_{\varphi_{0}}$. Consequently, we have

$$
\int|\nabla u|_{\varphi}^{2}=\int\left|u_{, \bar{\alpha} \bar{\beta}}\right|_{\varphi}^{2}=0
$$

which implies $u=0$.

Now we return to the proof of Lemma 4.2

Proof of Lemma 4.2 Suppose $\left(f_{i}, s_{i}\right) \in \mathcal{R}(T)$ such that $f_{i}$ converges strongly in $C^{\alpha}(M)$ to $f$ and $s_{i} \rightarrow$ $s$ as $i \rightarrow \infty$. We want to prove that $(f, s) \in \mathcal{R}(T)$. By definition, we may assume that $\left(f_{i}, s_{i}\right)=T\left(u_{i}, s_{i}\right)$ where $u_{i} \in C^{4, \alpha}(M)$. From the definition of the operator $T$, and since $T(u+C, s)=T(u, s)$, we can set

$$
\int u_{i} \omega_{\varphi_{0}}=0
$$

In other words,

$$
\mathcal{L}_{\left(\varphi_{0}, t_{0}\right)} u_{i}+s_{i}\left(R_{\varphi_{0}}-\underline{R}+\operatorname{tr}_{\varphi_{0}} \chi-\underline{\chi}\right)=\mathrm{f}_{\mathrm{i}}
$$

For any function $u \in C^{4, \alpha}(M)$, we have

$$
\begin{aligned}
\int u \mathcal{L}_{\left(\varphi_{0}, t_{0}\right)} u \omega_{\varphi_{0}}^{n} & =\int\left(-t_{0} \Delta_{\varphi_{0}}^{2} u-t_{0} u_{, \bar{\alpha} \beta}\left(\operatorname{Ric}_{\varphi_{0}}\right)_{\alpha \bar{\beta}}+\left(1-t_{0}\right) u_{, \bar{\alpha} \beta} \chi_{\alpha \bar{\beta}}\right) u \omega_{\varphi_{0}}^{n} \\
& =\int\left(-t_{0}\left|u_{, \bar{\alpha} \bar{\beta}}\right|_{\varphi_{0}}^{2}-\left(1-t_{0}\right) u_{, \bar{\alpha}} u_{, \beta} \chi_{\alpha \bar{\beta}}+u_{, \bar{\delta}}\left(t_{0} R_{\varphi_{0}}-\left(1-t_{0}\right) \operatorname{tr}_{\varphi_{0}} \chi\right)_{, \delta} \mathrm{u}\right) \omega_{\varphi_{0}}^{\mathrm{n}}
\end{aligned}
$$

Since $F\left(\varphi_{0}, t_{0}\right)=\left(0, t_{0}\right)\left(t_{0}<1\right)$ and $\chi>0$, we have

$$
-\int u \mathcal{L}_{\left(\varphi_{0}, t_{0}\right)} u \omega_{\varphi_{0}}^{n} \geq \epsilon_{0} \int\left|\nabla_{\varphi_{0}} u\right|_{\varphi_{0}}^{2} \omega_{\varphi_{0}}^{n}
$$

Therefore, we have

$$
\begin{aligned}
\epsilon_{0} \int\left|u_{i}\right|^{2} \omega_{\varphi_{0}}^{n} & \leq C \cdot \epsilon_{0} \int\left|\nabla_{\varphi_{0}} u_{i}\right|^{2} \omega_{\varphi_{0}}^{n} \\
& \leq C \epsilon \int\left(s_{i}\left(R_{\varphi_{0}}-\underline{R}+\operatorname{tr}_{\varphi_{0}} \chi-\underline{\chi}\right)-\mathrm{f}_{\mathrm{i}}\right) \mathrm{u}_{\mathrm{i}} \omega_{\varphi_{0}}^{\mathrm{n}} \\
& \leq \frac{1}{2} \epsilon_{0} \int\left|u_{i}\right|^{2} \omega_{\varphi_{0}}^{n}+C
\end{aligned}
$$


Thus,

$$
\int\left|u_{i}\right|^{2} \omega_{\varphi_{0}}^{n} \leq C
$$

It follows that

$$
\int\left|\nabla_{\varphi_{0}} u_{i}\right|^{2} \omega_{\varphi_{0}}^{n} \leq C
$$

Let's first consider the case when $0<t_{0}<1$. Given the various bounds above, it's not hard to prove that

$$
\int\left|\Delta_{\varphi_{0}} u_{i}\right|^{2} \omega_{\varphi_{0}}^{n} \leq C
$$

or

$$
\left\|u_{i}\right\|_{W_{\varphi_{0}}^{2,2}}^{2}<C .
$$

Now we can re-write the equation for $u_{i}$ as

$$
t_{0} \Delta_{\varphi_{0}}\left(\Delta_{\varphi_{0}} u_{i}\right)=-t_{0} u_{i, \bar{\alpha} \beta}\left(\operatorname{Ric}_{\varphi_{0}}\right)_{\alpha \bar{\beta}}+\left(1-t_{0}\right) u_{i, \bar{\alpha} \beta} \chi_{\alpha \bar{\beta}}+s_{i}\left(R_{\varphi_{0}}-\underline{R}+\operatorname{tr}_{\varphi_{0}} \chi-\underline{\chi}\right)-\mathrm{f}_{\mathrm{i}} .
$$

Note that the right hand side is uniformly bounded in $L^{2}$ space. Thus, we have

$$
\left\|\Delta_{\varphi_{0}} u_{i}\right\|_{W_{\varphi_{0}}^{2,2}}^{2}<C
$$

or

$$
\left\|u_{i}\right\|_{W_{\varphi_{0}}^{4,2}}^{2}<C .
$$

Following the standard bootstrapping argument in elliptic theory, we have

$$
\left\|u_{i}\right\|_{C^{4, \alpha}(M)} \leq C\left(\left\|u_{i}\right\|_{L^{2}(M)}+\left\|f_{i}-s_{i}\left(R_{\varphi_{0}}-\underline{R}+\operatorname{tr}_{\varphi_{0}} \chi-\underline{\chi}\right)\right\|_{\mathrm{C}^{\alpha}(\mathrm{M})}\right) \leq \mathrm{C}
$$

It follows that for any $\alpha^{\prime}<\alpha$, we can choose a subsequence $u_{i} \stackrel{C^{4, \alpha^{\prime}}(M)}{\longrightarrow} u$, as $i \rightarrow \infty$ and $u \in C^{4, \alpha}(M)$. Let $\left(f^{\prime}, s\right)=T(u, s)$, then $f_{i} \stackrel{C^{\alpha^{\prime}}(M)}{\longrightarrow} f^{\prime}$, as $i \rightarrow \infty$. Therefore, $(f, s)=\left(f^{\prime}, s\right)=T(u, s) \in \mathcal{R}(T)$. So we proved that $\mathcal{R}(T)$ is closed.

Following the standard theory on linear operators among Hilbert spaces, we have

Corollary 4.4 The following decomposition holds:

$$
C^{\alpha}(M) \times \mathbb{R}=\mathcal{R}(T) \oplus \mathbb{R}
$$

Proof By definition of the operator $T$, we know that for any $(f, s) \in \mathcal{R}(T)$, we have $\int_{M} f \omega_{\varphi_{0}}^{n}=0$. Set $C_{0}^{\alpha}(M)=\left\{f \in C^{\alpha}(M): \int_{M} f \omega_{\varphi_{0}}^{n}=0\right\}$. Then, $\mathcal{R}(T) \subset C_{0}^{\alpha}(M) \times \mathbb{R}$. The equality holds since $T$ is a linear, self adjoint operator. So the dimension of Kernel is the same as the dimension of coKernal. Since $\operatorname{dim} \operatorname{Ker} T=1$, thus $\operatorname{dim} \operatorname{coKer} T=1$. It follows that

$$
C^{\alpha}(M) \times \mathbb{R}=\mathcal{R}(T) \oplus \mathbb{R}
$$

Denote $\tilde{\mathcal{R}}(T)=\pi_{C^{\alpha}(M)} \mathcal{R}(T)=C_{0}^{\alpha}(M) \times \mathbb{R}$.

Proof of Theorem 4.1 Without loss of generality, we can assume $\int_{M} \varphi_{0} \omega_{\varphi_{0}}^{n}=0$. Consider $F^{1}(\varphi, t)=$ $\pi_{C^{\alpha}(M)} F(\varphi, t)$ as the projection of $F$ to the $C^{\alpha}(M)$ component. Thus

$$
F^{1}(\varphi, t)=t\left(R_{\varphi}-\underline{R}\right)-(1-t)\left(\operatorname{tr}_{\varphi} \chi-\underline{\chi}\right) .
$$


Consider the map

$$
\begin{aligned}
\Psi: \mathcal{H}^{4, \alpha}(M) \times[0,1] & \longrightarrow\left(C_{0}^{\alpha}(M) \oplus \mathbb{R}\right) \times[0,1] \\
(\varphi, t) & \longmapsto\left(\tilde{\pi} \circ F^{1}(\varphi, t)+\int \varphi \omega_{\varphi_{0}}^{n}, t\right),
\end{aligned}
$$

where $\tilde{\pi}$ is the projection to $C_{0}^{\alpha}(M)$, i.e. $\tilde{\pi}(f)=f-\oint_{M} f \omega_{\varphi_{0}}^{n}$ for any function $f \in C^{\alpha}(M)$. Note that

$$
\oint_{M} F^{1}\left(\varphi, t_{0}\right) \omega_{\varphi_{0}}^{n}=0
$$

Thus, corresponding to the variation $\delta \varphi=u$, the variation of $\tilde{\pi} \circ F^{1}$ at $t=t_{0}$ is:

$$
\left.\delta\left(\tilde{\pi} \circ F^{1}\right)(\varphi, t)\right|_{t=t_{0}}=\mathcal{L}_{\left(\varphi_{0}, t_{0}\right)} u+s\left(R_{\varphi_{0}}-\underline{R}+\operatorname{tr}_{\varphi_{0}} \chi-\underline{\chi}\right) .
$$

It follows that

$$
\begin{aligned}
\left.\mathcal{D} \Psi\right|_{\left(\varphi_{0}, t_{0}\right)}(u, s) & =\left(\left.\mathcal{D} F^{1}\right|_{\varphi_{0}, t_{0}}(u, s)+\int u \omega_{\varphi_{0}}^{n}, s\right) \\
& =\left(\mathcal{L}_{\left(\varphi_{0}, t_{0}\right)} u+s\left(R_{\varphi_{0}}-\underline{R}+\operatorname{tr}_{\varphi_{0}} \chi-\underline{\chi}\right)+\int \mathrm{u} \omega_{\varphi_{0}}^{\mathrm{n}}, \mathrm{s}\right) .
\end{aligned}
$$

By our discussions earlier, $\left.\mathcal{D} \Psi\right|_{\left(\varphi_{0}, t_{0}\right)}: C^{4, \alpha}(M) \times \mathbb{R} \rightarrow\left(C_{0}^{\alpha}(M) \oplus \mathbb{R}\right) \times \mathbb{R}$ is bijective. Thus, by inverse function theorem, we can find its inverse

$$
\Psi^{-1}:\left(C_{0}^{\alpha}(M) \oplus \mathbb{R}\right) \times[0,1] \rightarrow C^{4, \alpha}(M) \times[0,1]
$$

near $\left(0, t_{0}\right)$. In other words, there exists a small open neighborhood

$$
V_{0, t_{0}} \subset C^{\alpha}(M) \times[0,1]=\left(C_{0}^{\alpha}(M) \oplus \mathbb{R}\right) \times[0,1]
$$

where $\Psi^{-1}$ is well defined such that

$$
\Psi^{-1}\left(V_{0, t_{0}}\right)=U_{\left(\varphi_{0}, t_{0}\right)} \subset C^{4, \alpha}(M) \times[0,1] .
$$

Denote

$$
\begin{aligned}
\tilde{F}=F \circ \Psi^{-1}: V_{\left(0, t_{0}\right)} & \longrightarrow\left(C_{0}^{\alpha}(M) \oplus \mathbb{R}\right) \times[0,1] \\
(w+a, t) & \longmapsto\left(w+\int_{M} F^{1} \circ \Psi^{-1}(w+a, t) \omega_{\varphi_{0}}^{n}, t\right) .
\end{aligned}
$$

and

$$
f(w, a, t)=\int_{M} F^{1} \circ \Psi^{-1}(w+a, t) \omega_{\varphi_{0}}^{n} .
$$

Then the linearized operator is

$$
\begin{aligned}
\left.\mathcal{D} \tilde{\mathcal{F}}\right|_{(w+a, t)}:\left(C_{0}^{\alpha}(M) \oplus \mathbb{R}\right) \times \mathbb{R} & \longrightarrow\left(C_{0}^{\alpha}(M) \oplus \mathbb{R}\right) \times \mathbb{R} \\
(u+b, s) & \longmapsto\left(u+\left(\frac{\partial f}{\partial w}(w, a, t) u+\frac{\partial f}{\partial a}(w, a, t) b+\frac{\partial f}{\partial t}(w, a, t) s\right), s\right) .
\end{aligned}
$$

Next we consider its kernel $\operatorname{Ker}\left(\left.\mathcal{D} \tilde{\mathcal{F}}\right|_{(w+a, t)}\right)$. Since $\tilde{F}=F \circ \Psi^{-1}$ and $\Psi$ is bijection, we need to consider the $\operatorname{dim} \operatorname{Ker}\left(\left.\mathcal{D} \mathcal{F}\right|_{(\varphi, t)}\right)$. Clearly, we have

$$
\operatorname{Ker}\left(\left.\mathcal{D F}\right|_{(\varphi, t)}\right)=\left\{(u, 0) \in C^{4, \alpha}(M) \times \mathbb{R} \mid \mathcal{L}_{(\varphi, t)} u=0\right\} .
$$


Following the proof of the previous lemma, we can easily prove that for any $(u, 0) \in \operatorname{Ker}\left(\left.\mathcal{D F}\right|_{(\varphi, t)}\right)$, we have

$$
u=\oint_{M} u \omega_{\varphi_{0}}^{n}
$$

On the other hand, it is clear that

$$
\left\{(C, 0) \in C^{4, \alpha}(M) \times \mathbb{R}\right\} \subset \operatorname{Ker}\left(\left.\mathcal{D} \mathcal{F}\right|_{(\varphi, t)}\right) .
$$

Therefore, $\operatorname{dim} \operatorname{Ker}\left(\left.\mathcal{D} \mathcal{F}\right|_{(\varphi, t)}\right)=1$. It follows that $\operatorname{dim}_{\mathbb{R}} \operatorname{Ker}\left(\left.\mathcal{D} \tilde{\mathcal{F}}\right|_{(w+a, t)}\right)=1$ for $(w+a, t)$ sufficiently close to $\left(0, t_{0}\right)$. We claim that $\frac{\partial f}{\partial a}(w, a, t)=0$. Otherwise, $\operatorname{dim}_{\mathbb{R}} \operatorname{Ker}\left(\left.\mathcal{D} \tilde{\mathcal{F}}\right|_{(w+a, t)}\right)=0$. Note for any $(u+b, s) \in \operatorname{Ker}\left(\left.\mathcal{D} \tilde{\mathcal{F}}\right|_{(w+a, t)}\right.$, we have

$$
\left(u+\left(\frac{\partial f}{\partial w}(w, a, t) u+\frac{\partial f}{\partial a}(w, a, t) b+\frac{\partial f}{\partial t}(w, a, t) s\right), s\right)=(0+0,0) .
$$

It follows that $u=s=0$ and

$$
\frac{\partial f}{\partial a}(w, a, t) b=0
$$

If $\frac{\partial f}{\partial a}(w, a, t) \neq 0$, then $b=0$. It follows that $\left.\operatorname{Ker}(\mathcal{D} \tilde{\mathcal{F}})\right|_{(w+a, t)}=0$. This is a contradiction so our claim holds. It follows that $f(w, a, t)=f(w, t)$. Therefore,

$$
\tilde{F}(w+a, t)=(w+f(w, t), t)
$$

We want to find the preimage of $\tilde{F}$ for $(0, t)$. We claim that $f(0, t)=0$. First, we look at $(\varphi, t)=$ $\Psi^{-1}(0, a, t)$. It means that

$$
\Psi(\varphi, t)=(0, a, t)
$$

In other words, we have

$$
\left(\tilde{\pi} \circ F^{1}(\varphi, t)+\int_{M} \varphi \omega_{\varphi_{0}}^{n}, t\right)=(0, a, t) .
$$

It follows that $\int_{M} \varphi \omega_{\varphi_{0}}^{n}=a$ and $\tilde{\pi} \circ F^{1}(\varphi, t)=0$. It follows that

$$
F^{1}(\varphi, t)-\int_{M} F^{1}(\varphi, t) \omega_{\varphi_{0}}^{n}=0
$$

which implies that $F^{1}(\varphi, t)(x) \equiv C$. Note that $\int_{M} F^{1}(\varphi, t) \omega_{\varphi}^{n}=0$ by definition. Consequently, $F^{1}(\varphi, t)(x) \equiv 0$, and then

$$
f(0, t)=\int_{M} F^{1}(\varphi, t) \omega_{\varphi_{0}}^{n}=0 .
$$

So

$$
\tilde{F}(0, a, t)=(0+f(0, t), t)=(0, t) .
$$

We therefore have $F\left(\Psi^{-1}(0, a, t)\right)=(0, t)$. This completes the proof.

\section{$5 \quad$ Twisted K-stability}

\subsection{Twisted K-stability}

Corresponding to the twisted K-energy functional, for any test configuration $\lambda$ of $M$ (see [81] for a precise definition in the algebraic case when $\Omega$ represents the first Chern class of an holomorphic line bundle $L$, roughly speaking, it means that $M$ is embedded in $\mathbb{P}^{N_{k}}$ by the linear system $|-k L|$ and 
$\lambda: \mathbb{C}^{*} \rightarrow G L\left(N_{k}, \mathbb{C}\right)$ is a one parameter subgroup ) we could define twisted Futaki invariant. Suppose $\lambda(s)=s^{A}$, define a function on $\mathbb{P}^{N_{k}}$,

$$
h_{A}=\frac{z A z^{*}}{z z^{*}}
$$

then we make the following definition:

\section{Definition 5.1 (twisted Futaki invariant)}

$$
F u t_{t}(\Omega, \chi ; \lambda)=(1-t) F u t(\chi ; \lambda)+t F u t(\Omega ; \lambda)
$$

where Fut $(\Omega ; \lambda)$ is the algebraically defined Donaldson-Futaki invariant of $\lambda$, and

$$
\begin{aligned}
\operatorname{Fut}(\chi ; \lambda) & =\operatorname{Ch}(\chi ; \lambda)-\underline{\chi} \operatorname{Ch}(M ; \lambda) \\
& =\lim _{t \rightarrow 0}\left\{\int_{M_{s}} h_{A} \lambda\left(s^{-1}\right)^{*} \chi \wedge\left(\left.\frac{1}{k} \omega_{F S}\right|_{M_{s}}\right)^{[n-1]}-\underline{\chi} \int_{M_{s}} h_{A}\left(\left.\frac{1}{k} \omega_{F S}\right|_{M_{s}}\right)^{[n]}\right\} \\
& =\lim _{t \rightarrow 0}\left\{\int_{M} \chi \wedge \lambda(s)^{*}\left(h_{A} \omega_{s}^{[n-1]}\right)-\underline{\chi} \int_{M} \lambda(s)^{*}\left(h_{A} \omega_{s}^{[n]}\right)\right\}
\end{aligned}
$$

Remark 5.2 In the special case when the central fiber $M_{0}=\lim _{t \rightarrow 0} \lambda(s) M$ is a normal variety, Fut $t_{t}(\Omega, \chi ; \lambda)$ reduces to the usual log-Futaki invariant of the log pair $\left(M_{0},(1-t) D_{0}\right)$ (first introduced by [52] for the study of conical Kähler-Einstein metrics) where $D_{0}=\lim _{s \rightarrow 0} \lambda(s) D$ for a generic divisor $D$ in the linear system $\left|L_{\chi}\right|$.

If $\chi$ and $\omega$ both belongs to $2 \pi C_{1}(M)$, the twisted cscK metrics equation reduces to the Aubin's continuity path, for which the Futaki's invariant is already explicitly defined, see [81, Formula (4.10)].

Definition 5.3 (twisted K-semistable/stable) A triple $(\Omega, \chi, s)$ as above is called twisted K-semistable if

$$
\text { Fut }_{t}(\Omega, \chi ; \lambda) \leq 0
$$

for any test configuration $\lambda$; and it is called twisted K-stable if

$$
F u t_{t}(\Omega, \chi ; \lambda)<0
$$

for any $\lambda$ nontrivial.

It follows from the definition that $\operatorname{Fut}(\chi ; \lambda)$ depends linearly on $\chi$ for any fixed $\lambda$. It is proved in 81 , Thm 6] that

$$
\operatorname{Fut}(\chi ; \lambda) \leq \operatorname{Fut}(D ; \lambda)
$$

for any divisor $D$ in the linear system of $L_{\chi}$, and for any fixed test configuration $\lambda$ the equality holds for generic element $D$ in this linear system. One consequence is that $\operatorname{Fut}(\chi ; \lambda)$ is independent of the particular choice of $\chi$, therefore the notion of twisted $K$-stability for a triple $(\Omega, \chi, s)$ is independent of the choice of $\chi$ in a fixed cohomology class also .

Proposition 5.4 (Linear Interpolation) If $\left(\Omega, \chi, t_{0}\right)$ and $\left(\Omega, \chi, t_{1}\right)$ are both twisted $K$-semistable, and one of them is twisted $K$-stable, then $(\Omega, \chi, t)$ is twisted $K$-stable for any $t \in\left(t_{0}, t_{1}\right)$.

Proof Since $\operatorname{Fut}_{t}(\Omega, \chi ; \lambda)$ depends on $t$ linearly for any fixed test configuration $\lambda$, the twisted K-stability for the two parameters $t_{0}$ and $t_{1}$ implies a preferred sign of Fut $t_{t}$ for any $t$ in between.

Conjecture 5.5 If $(\Omega, \chi, t)$ with $t<1$ is twisted K-stable iff Equation (1.1) admits a solution for the parameter $t$. 
It should be noticed that the twisted $\csc K$ equation (Equation (1.1) for $t=\frac{1}{2}$, the middle point of our continuous path) was already studied by [56, 77. Stoppa in [77, Theorem 1.3] gave a cohomological obstruction, Kähler slope stability, to the existence extending the result of Ross-Thomas 72 for the untwisted case. In another direction, [64, Conjecture 1] conjectured a numerical criterion for the existence of solution to Equation (1.3) (Equation (1.1) for $t=0$, the starting point of our continuous path), based on the study of deformation to the normal cone, a particular type of test configuration, extensively used in the work [72]. And recently this conjecture was proved in [41, Theorem 1.3] for toric manifolds, therefore our conjecture here holds on toric manifolds for the starting point.

Remark 5.6 While we were preparing this note, we noticed the recent work by R. Dervan [44] who introduced also the notion of (uniform) twisted K-stability and proved that the existence of twisted cscK metric implies the (uniform) twisted K-stability by using the lower bound of the twisted Calabi functional.

\section{Twisting cscK metric with higher degree form}

While a $(1,1)$-form $\chi>0$ is convenient, from PDE point of view, there is no particular reasons to restrict oneselves to this setting only. For any integer $k \in\{1, \cdots, n\}$, consider a closed $(k, k)$-form $\mu_{k}=\chi^{k}$, one can define a new functional

$$
\frac{d J_{\mu_{k}}}{d t}=\int_{M} \dot{\varphi}\left(\mu_{k} \wedge \omega_{\varphi}^{n-k}-c_{k} \omega_{\varphi}^{n}\right)
$$

where the constant

$$
c_{k}=\frac{\left[\mu_{k}\right] \cdot[\omega]^{n-k}}{[\omega]^{n}}
$$

Remark that the closedness of $\mu_{k}$ guarantee that $J_{\mu_{k}}$ is well-defined. Note that this functional has been studied in the literature before (cf. [55]). The Euler-Lagrange equation is

$$
\frac{\mu_{k} \wedge \omega_{\varphi}^{n-k}}{\omega_{\varphi}^{n}}=c_{k}
$$

When $k=0$, this is the well-known $I$ functional. When $k=1$, then this is just the usual $J$ functional we discussed here. When $k=n$, then $\mu$ is a volume form, for which the Euler-Lagrange equation reduces to

$$
\omega_{\varphi}^{n}=\frac{1}{c_{n}} \mu_{n}
$$

which is a standard complex Monge-Ampère equation. In fact, this is precisely the Calabi's volume form conjecture. There is a simple observation for this family of functionals in a special case, where $\mu_{k}=\omega_{0}^{k}, k=1, \cdots, n$ for some Kähler metric $\omega_{0}$.

Proposition 6.1 For any $\phi \in \mathcal{H}_{\omega_{0}}$,

$$
J_{\omega_{0}^{n}}(\phi) \geq J_{\omega_{0}^{n-1}}(\phi) \geq \cdots \geq J_{\omega_{0}}(\phi) \geq \frac{1}{n+1} J(\phi)
$$

Proof If suffices to prove $J_{\omega_{0}^{k+1}}(\phi) \geq J_{\omega_{0}^{k}}(\phi)$. However, this is almost obvious by the definition. Take the standard linear path $\phi_{t}=t \phi$, then 


$$
\begin{aligned}
J_{\omega_{0}^{k}}(\phi) & =\int_{0}^{1} d t \int_{M} \dot{\phi}_{t}\left(\omega_{0}^{k}-\omega_{\phi_{t}}^{k}\right) \omega_{\phi_{t}}^{n-k} \\
& =\int_{0}^{1} d t \int_{M} \phi t\left(\omega_{0}-\omega_{\phi}\right)\left(\omega_{0}^{k-1}+\omega_{0}^{k-2} \omega_{t \phi}+\cdots+\omega_{t \phi}^{k-1}\right) \omega_{t \phi}^{n-k} \\
& =\int_{0}^{1} t d t \int_{M} \sqrt{-1} \partial \phi \wedge \bar{\partial} \phi \wedge\left(\omega_{0}^{k-1} \omega_{t \phi}^{n-k}+\omega_{0}^{k-2} \omega_{t \phi}^{n-k+1}+\cdots+\omega_{t \phi}^{n-1}\right)
\end{aligned}
$$

Since each integrand is a positive term, $J_{\omega_{0}^{k+1}} \geq J_{\omega_{0}^{k}}$. And the last inequality $J_{\omega_{0}} \geq \frac{1}{n+1} J$ is wellknown (cf. Bando-Mabuchi[3, Eq. 1.6.4]).

Similar to the case $k=1$, there is also a moment map picture associated with this functional.

One can also define a new continuity path as

$$
t\left(R_{\varphi}-\underline{R}\right)=(1-t)\left(\frac{\mu_{k} \wedge \omega_{\varphi}^{n-k}}{\omega_{\varphi}^{n}}-c_{k}\right), \quad \forall t \in[0,1]
$$

This is the Euler-Lagrange equation of the twisted K-energy functional

$$
E_{\mu_{k}, t}=t E+(1-t) J_{\mu_{k}} .
$$

Then, one can formulate twisted K-stability and other geometric notions similarly. It is straightforward to prove the following

Theorem 6.2 For any closed positive $\chi>0$, the twisted $K$-energy functional $E_{\mu_{k}, t}$ is convex along $C^{1,1}$ geodesic segment for any $k=1,2, \cdots, n$.

Following proof in Section 3, we have

Theorem 6.3 For any $k>0$, the twisted cscK metric equation is open for any $t \in[0,1)$.

Theorem 6.4 If $(M,[\omega])$ is $K$-semistable and $K$-stable for $J_{\mu_{k}}$, then the twisted trip structure $\left(M,[\omega], \mu_{k}, t\right)$ is $K$-stable for $t \in[0,1)$.

Moreover, we can ask question parallel to those in previous sections. To avoid redundancy, we only list the following conjecture

Conjecture 6.5 There exists a twisted cscK metric if and only if the corresponding twisted K-energy functional is proper in terms of geodesic distance.

Here we give a proof of the necessary part for $k=n$ and $t \in(0,1)$.

Proof Suppose $\omega_{\varphi}$ is a twisted cscK metric for $\mu>0$ an positive volume form and $t_{0} \in(0,1)$, then there exists a small $\delta>0$ such that the twisted cscK metric equation can be solved for any $t^{\prime} \in\left(t_{0}, t_{0}+2 \delta\right) \subset$ $(0,1)$. Fix $t^{\prime}=t_{0}+\delta$. Then,

$$
t^{\prime} E+\left(1-t^{\prime}\right) J_{\mu}
$$

is bounded from below.

Firstly, by Yau's solution of Calabi's Volume form Conjecture, there exists a unique Kähler metric $\omega_{0} \in[\omega]$ such that

$$
\mu=c_{n} \omega_{0}^{n}
$$


which implies $J_{\mu}=c_{n} J_{\omega_{0}^{n}} \geq \frac{c_{n}}{n+1} J$ by Prop. 6.1]

Now

$$
\begin{aligned}
t_{0} E+\left(1-t_{0}\right) J_{\mu} & =\frac{t_{0}}{t^{\prime}}\left(t^{\prime} E+\left(1-t_{0}\right) \frac{t^{\prime}}{t_{0}} J_{\mu}\right) \\
& \left.=\frac{t_{0}}{t^{\prime}}\left(t^{\prime} E+\left(1-t^{\prime}\right) J_{\mu}\right)+\frac{t_{0}}{t^{\prime}}\left(\left(1-t_{0}\right) \frac{t^{\prime}}{t_{0}}-\left(1-t^{\prime}\right)\right) J_{\mu}\right) \\
& =\frac{t_{0}}{t^{\prime}}\left(t^{\prime} E+\left(1-t^{\prime}\right) J_{\mu}\right)+\frac{t_{0}}{t^{\prime}}\left(\frac{t^{\prime}}{t_{0}}-1\right) J_{\mu} \\
& =\frac{t_{0}}{t^{\prime}}\left(t^{\prime} E+\left(1-t^{\prime}\right) J_{\mu}\right)+\frac{t^{\prime}-t_{0}}{t^{\prime}} J_{\mu} \\
& \geq \frac{t_{0}}{t^{\prime}}\left(t^{\prime} E+\left(1-t^{\prime}\right) J_{\mu}\right)+\frac{\delta}{\delta+t_{0}} J_{\mu} .
\end{aligned}
$$

Now the first term is bounded from below and the second is proper, thus the twisted K-energy $t_{0} E+$ $\left(1-t_{0}\right) J_{\mu}$ is proper in terms of the geodesic distance. 


\section{References}

[1] C. Arezzo, F. Pacard, Blowing up and desingularizing constant scalar curvature Kähler manifolds. Acta Math. 196 (2006), no. 2, 179-228.

[2] C. Arezzo, F. Pacard and M. Singer: Extremal metrics on blowups. Duke Math. J. 157 (2011), no. $1,1-51$.

[3] S. Bando and T.Mabuchi: Uniquness of Einstein Kähler metrics modulo connected group actions. In Algebraic Geometry, Advanced Studies in Pure Math., $198 \%$.

[4] E.D. Bedford and T.A. Taylor: The Dirichlet problem for the complex Monge-Ampère operator. Invent. Math., 37:1-44, 1976.

[5] R.J. Berman: K-polystability of $\mathbb{Q}$-Fano varieties admitting Kähler-Einstein metrics. Preprint, http://arxiv.org/abs/1205.6214.

[6] R. J. Berman: A thermodynamical formalism for Monge-Ampé re equations, Moser-Trudinger inequalities and Kähler Einstein metrics, Adv. Math. 248 (2013), 1254?1297.

[7] R.J. Berman, B. Berndtsson: Convexity of the K-energy on the space of Kähler metrics. Preprint, http://arxiv.org/abs/1405.0401

[8] Z. Blocki: On the regularity of the complex Monge-Ampère operator, Contemporary Mathematics 222, Complex Geometric Analysis in Pohang, ed. K.-T.Kim, S.G.Krantz, pp.181-189, Amer. Math. Soc. 1999.

[9] Z. Blocki: Interior Regiularity of the complex Monge-Ampère equation in complex domains Duke Math. Jour., vol 105, no. 1, 167-181, 2000.

[10] L.A. Caffarelli: Interior $W^{2, p}$ estimates for solutions to the Monge-Ampére equation, Annals of Math. 131, 135-150, 1990

[11] L.A. Caffarelli and C.E. Gutiérrez: Properties of the solutions of the linearised Monge-Ampére equation, Amer. Jour. Math. 119 423-465 1997

[12] L.A. Caffarelli, L. Nirenberg and J. Spruck: The Dirichlet problem for nonlinear second-order elliptic equation I. Monge-Ampère equation. Comm. on pure and appl. math., XXXVII:369-402, 1984.

[13] L.A. Caffarelli, J.J. Kohn, L. Nirenberg, and J. Spruck: The Dirichlet problem for nonlinear second-order elliptic equation II. complex Monge-Ampère equation, and Uniformly Elliptic equations. Comm. on Pure and Appl. math., 38:209-252, 1985.

[14] E. Calabi: Extremal Kähler metrics. In Seminar on Differential Geometry, volume 16 of 102, pages 259-290. Ann. of Math. Studies, University Press, 1982.

[15] E. Calabi: Extremal Kähler metrics, II. In Differential geometry and Complex analysis, pages 96-114. Springer, 1985.

[16] X.-X. Chen, L. Li and M. Paun: Approximation of weak geodesics and subharmonicity of Mabuchi energy Preprint, http://arxiv. org/abs/1409.7896.

[17] X.-X. Chen: Space of Kähler metrics. Journal of Differential Geometry, 56(2):189-234, 2000. 
[18] X.-X. Chen: A new parabolic flow in Kähler manifolds. Comm. Anal. Geom. 12 (2004), no. 4, 837 ? 852 .

[19] X.-X. Chen: On the lower bound of the Mabuchi energy and its application. Internat. Math. Res. Notices 2000, no. 12, 607-623.

[20] X.-X. Chen and G. Tian: Foliation by holomorphic discs and its application in Kähler geometry, 2003. Publ. Math. Inst. Hautes tudes Sci. No. 107 (2008), 1-10\%.

[21] X.-X. Chen: Space of Kähler metric (III) - Lower bound of the Calabi energy. Invent. Math. 175 (2009), no. 3, 453?503.

[22] X.-X. Chen: Space of Kähler metric (IV)-On the lower bound of the K-energy. http://arxiv.org/abs/0809.4081.

[23] X.-X. Chen and W.-Y. Ding Ricci flow on surfaces with degenerate initial metrics. J. Partial Differential Equations 20 (2007), no. 3, 193?202.

[24] X.-X. Chen. G. Tian, Z. Zhang: On the weak Kähler-Ricci flow. Trans. Amer. Math. Soc. 363 (2011), no. 6, 2849?2863.

[25] X.-X. Chen, S. Donaldson and S. Sun: Kähler-Einstein metrics on Fano manifolds. I: Approximation of metrics with cone singularities.. J. Amer. Math. Soc. 28 (2015), pp. 183-197 (I).

[26] X.-X. Chen, S. Donaldson and S. Sun: Kähler-Einstein metrics on Fano manifolds. II: Limits with

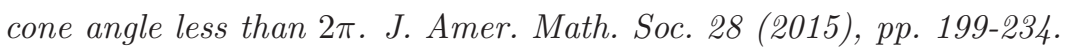

[27] X.-X. Chen, S. Donaldson and S. Sun: Kähler-Einstein metrics on Fano manifolds. III: Limits as cone angle approaches $2 \pi$ and completion of the main proof. J. Amer. Math. Soc. 28 (2015), pp. 235-278.

[28] X.-X. Chen and W-Y. He. On the Calabi flow, Amer. J. Math. 130 (2008), no. 2, 539-570.

[29] X.-X. Chen, C. LeBrun and B. Weber: On conformally Khler, Einstein manifolds. J. Amer. Math. Soc. 21 (2008), no. 4, 1137?1168

[30] X.-X. Chen and S. Sun: Space of Kähler metrics (V)-Kähler quantization. Metric and differential geometry, 19?41, Progr. Math., 297, Birkhuser/Springer, Basel, 2012.

[31] X.-X. Chen and S. Sun: Calabi flow, Geodesic rays, and uniqueness of constant scalar curvature Kähler metrics. Ann. of Math. (2) 180 (2014), no. 2, 407-454.

[32] X.-X. Chen and B. Wang: Space of Ricci flows (I). Comm. Pure Appl. Math. 65 (2012), no. 10, 1399-145\%.

[33] X.-X. Chen and B. Wang: Space of Ricci flows (II). Preprint, http://arxiv. org/abs/1405.6797, submitted.

[34] X.-X. Chen and B. Wang: Kähler Ricci flow on Fano manifolds(I). J. Eur. Math. Soc. (JEMS) 14 (2012), no. 6, 2001-2038.

[35] X.-X. Chen and Y.-Q. Wang: Bessel Functions, Heat Kernel and the Conical Kähler-Ricci Flow, Preprint, http://arxiv.org/abs/1305.0255.

[36] X.-X. Chen and Y.-Q. Wang: On the long time behaviour of the Conical Kähler- Ricci flows , Preprint, http://arxiv. org/abs/1402.6689. 
[37] X.-X. Chen and Y.-Q. Wang: On the regularity problem of complex Monge-Ampère equations with conical singularities, Preprint, http://arxiv.org/abs/1405.1021.

[38] X.-X. Chen and B. Weber: Moduli spaces of critical Riemannian metrics with $L^{n / 2}$ norm curvature bounds. Adv. Math. 226 (2011), no. 2, 1307-1330.

[39] X.-X. Chen, M. Paun and Y. Zeng: On deformation of extremal metrics, http://arxiv.org/abs/1506.01290.

[40] R.R. Coifman and S. Semmes: Interpolation of Banach Spaces, Perron Processes, and Yang-Mills. Amer. J. Math., 115(2):243-278, 1993.

[41] T.Collins, G.Székelyhidi: Convergence of the J-Flow on Toric manifolds. Preprint, http://arxiv.org/abs/1412. 4809

[42] T. Darvas: Envelopes and Geodesics in Spaces of Kähler Potentials. Preprint, http://arxiv.org/abs/1401.7318.

[43] T. Darvas: The Mabuchi Geometry of Finite Energy Classes. Preprint, http://arxiv. org/abs/1409.2072.

[44] R. Dervan, Uniform Stability of twisted constant scalar curvature Kähler metrics. Preprint, http://arxiv.org/abs/1412.0648

[45] H. S. Do: Degenerate complex Monge-Ampère flows on strictly pseudoconvex domains, http://arxiv.org/abs/1501.07167

[46] S. K. Donaldson: Symmetric spaces, Kähler geometry and Hamiltonian dynamics. Amer. Math. Soc. Transl. Ser. 2, 196, pages 13-33, 1999. Northern California Symplectic Geometry Seminar.

[47] S. K. Donaldson: Scalar curvature and projective embeddings. I. J. Differential Geom. 59 (2001), no. 3, 479-522.

[48] S. K. Donaldson: Scalar curvature and stability of toric varieties, Jour. Differential Geometry 62, 289-349, 2002

[49] S. K. Donaldson: Extremal metrics on toric surfaces: a continuity method, J. Differential Geom. 79 (2008), no. 3, 389-432.

[50] S. K. Donaldson: Constant scalar curvature metrics on toric surfaces, Geom. Funct. Anal. 19 (2009), no. 1, 83-136.

[51] S. K. Donaldson: Lower bounds on the Calabi functional. J. Differential Geom. 70 (2005), no. 3, 453-472.

[52] S. K. Donaldson; Kähler metrics with cone singularities along a divisor, Essays in Mathematics and its Applications. 2012, 49-79.

[53] P. Eyssidieux, V. Guedj, A. Zeriahi Weak solutions to degenerate complex Monge-Ampère Flows I, Math. Annal. DOI 10.1007/s00208-014-1141-4

[54] P. Eyssidieux, V. Guedj, A. Zeriahi Weak solutions to degenerate complex Monge-Ampère Flows II http://arxiv.org/abs/1407.2504

[55] H. Fang, M. Lai, J. Song and B. Weinkove: The J-flow on Kähler surfaces: a boundary case. Anal. PDE 7 (2014), no. 1, 215-226. 
[56] J. Fine: Constant scalar curvature Kähler metrics on fibred complex surfaces. J. Differential Geom. 68 (2004), no. 3, 397-432.

[57] A. Futaki: An obstruction to the existence of Einstein Kähler metrics. Invent. Math. 73 (1983), no. 3, $437-443$.

[58] B. Guan: The Dirichlet problem for complex Monge-Ampère equations and regularity of the pluicomplex Green function. Comm. Ana. Geom., 6(4):687-703, 1998.

[59] V. Guedj, A. Zeriahi: Regularizing properties of the twisted Kähler-Ricci flow. http://arxiv.org/abs/1306.4089.

[60] T. D. Jeffres, R. Mazzeo, and Y. Rubinstein, Kähler-Einstein metrics with edge singularities. http://arxiv.org/abs/1105.5216v3.

[61] J. Pook: Twisted Calabi flow on Riemann surfaces, IMRN, p. 1-26, volume 2015, Issue 9.

[62] C. LeBrun, S. R. Simanca: Extremal Kähler metrics and Complex Deformation Theory, Geom. and Fun. Analysis, Vol. 4, No. 3 (1994), 298-336.

[63] C. LeBrun, M. Singer: Existence and deformation theory for scalar-flat Kähler metrics on compact complex surfaces, Invent. Math. 112 (1993), no. 2, 273-313.

[64] M. Lejmi and G. Székelyhidi: The J-flow and stability, Preprint, http://arxiv.org/abs/1309.2821.

[65] H.-Z. Li, B. Wang and K. Zheng: Regularity scales and convergence of the Calabi flow. Preprint, http://arxiv.org/abs/1501.01851.

[66] T. Mabuchi: Some symplectic geometry on compact Kähler manifolds I. Osaka, J. Math., 24:227252, $198 \%$.

[67] E. D. Nezza, C.-H. Lu: Uniqueness and short time regularity of the weak Kähler-Ricci flow, http://arxiv.org/abs/1411.7958.

[68] S. Paul: Stable Pairs and Coercive Estimates for The Mabuchi Functional, Preprint, http://arxiv. org/abs/1308.4377.

[69] S. Paul, G. Tian: CM stability and the generalized Futaki invariant II. Preprint, http://arxiv.org/abs/math/0606505.

[70] Pogorelov, A. V.: The Drichelet problem for the multidimensional analogue of the Monge-Ampère equation (Russian), Dokl. Akad. Nauk SSSR 201 (1971), 790-793.

[71] Pogorelov, A. V.: The Minkowski multidimensional problem, J. Wiley, New York, 1978

[72] J. Ross, R. Thomas: An obstruction to the existence of constant scalar curvature Kähler metrics. J. Differential Geom. 72(2006) no. 3, 429-466.

[73] Y. A. Rubinstein, S.Zelditch: Bergman approximations of harmonic maps into the space of Kähler metrics on toric varieties Priprint, http://arxiv. org/abs/0803.1249.

[74] S. Semmes: Complex Monge-Ampère equations and sympletic manifolds. Amer. J. Math., 114:495550, 1992. 
[75] J. Song and B. Weinkove: On the convergence and singularities of the J-flow with applications to the Mabuchi energy Comm. Pure Appl. Math. 61 (2008), no. 2, 210-229.

[76] J. Stoppa: K-stability of constant scalar curvature Kähler manifolds. Adv. Math. 221 (2009), no. 4, 1397-1408.

[77] J. Stoppa: Twisted constant scalar curvature Kähler metrics and Kähler slope stability. J. Differential Geom. 83 (2009), no. 3, 663-691.

[78] J. Streets: Long time existence of Minimizing Movement solutions of Calabi flow, http://arxiv. org/abs/1208.2718.

[79] G. Székelyhidi: Greatest Lower bound on the Ricci curvature of Fano manifolds, Compositio Math. 147(2011), 319-331.

[80] G. Székelyhidi: The partial $C^{0}$-estimate along the continuity method, Preprint, http://arxiv.org/abs/1310.8471.

[81] G. Székelyhidi: A remark on Conical Kähler-Einstein metrics, Math. Res. Lett. 20 (2013), no. 3, 581-590.

[82] G. Tian: On Calabi's conjecture for complex surfaces with positive first Chern class, Invent. Math. 101, no.1, 101-172.

[83] G. Tian, J. Viaclovsky: Moduli spaces of critical metrics in dimension four, Adv. Math., 196 (2005),346-372.

[84] G. Tian: Kähler-Einstein metrics with positive scalar curvature. Invent. Math. 130 (1997), no. 1, $1-3 \%$.

[85] G. Tian, S.-T. Yau: Kähler-Einstein metrics on complex surfaces with $C_{1}>0$, Comm. Math. Phys. 112 (1987), no. 1, 175-203.

[86] G. Tian, X.-H. Zhu: Convergence of Kähler-Ricci flow. J. Amer. Math. Soc. 20 (2007), no. 3, 675-699.

[87] N. S. Trudinger and X-J. Wang: The Monge-Ampère equation and its geometric applications, Handbook of Geometric analysis, International Press, 2008, Vol. I, pp. 467-524.

[88] Urbas, J. I. E.: Regularity of generalized solutions of Monge-Ampère equations, Math. Z. 197 (1988), 365-393.

[89] B. Wang: On the conditions to extend Ricci flow (II). Int. Math. Res. Not. IMRN 2012, no. 14 (2012): 3192?223.

[90] Y.-Q. Wang: Pseudolocality of the Ricci flow under integral bound of curvature. J. Geom. Anal. 23 (2013), no. 1, 1-23.

[91] Y. Wang: A remark on $C^{2, \alpha}$-regularity of the complex Monge-Amperre Equation, Preprint, http://arxiv.org/abs/1111.0902.

[92] B. Weinkove: Convergence of the J-flow on Kähler surfaces. Comm. Anal. Geom. 12 (2004), no. 4, 949-965.

[93] S.-T. Yau. On the Ricci curvature of a compact Kähler manifold and the complex Monge-Ampere equation, $I^{*}$. Comm. Pure Appl. Math., 31:339-441, 1978. 
[94] G. Tian. Some aspects of Kähler Geometry, 199\%. Lecture note taken by Meike Akeveld.

[95] B. Zhu and X.-H. Zhu Minimizing weak solutions for Calabi's extremal metrics on toric manifolds. Calc. Var. Partial Differential Equations 32 (2008), no. 2, 191-21\%. 